\title{
Transcatheter closure of multi-hole perimembranous ventricular septal defect with aneurysm using two occluders
}

\author{
Yi-yuan Huang, Zhen-fei Fang, Jian-jun Tang, Liang Tang, Xin-qun Hu, Sheng-hua Zhou \\ Department of Cardiology, Second Xiangya Hospital, Central South University, Changsha, Hunan, China
}

Adv Interv Cardiol 2017; 13, 1 (47): 75-77

DOI: https://doi.org/10.5114/aic.2017.66191

\begin{abstract}
A 33-year-old man presented to our department for recurrent respiratory tract infections since early childhood. His physical and mental development was normal. A grade 4/6 pansystolic murmur was heard over the left sternal border.

Transthoracic echocardiography (TTE) revealed a moderate-sized perimembranous ventricular septal defect (PmVSD) with a $14 \mathrm{~mm} \times 9 \mathrm{~mm}$ aneurysm (Figures $1 \mathrm{~A}, \mathrm{~B}$ ). The diameter of the exit was $5 \mathrm{~mm}$. The left atrium and ventricle were dilated. Their diameters were $35 \mathrm{~mm}$ and $55 \mathrm{~mm}$ respectively. There were mild mitral and tricuspid regurgitations. We attempted to perform a transcatheter closure of this PmVSD under general anesthesia with the guidance of fluoroscopy and echocardiography.

However, a left ventricle (LV) angiogram showed that the VSD was characterized by two exits with diameters of $7 \mathrm{~mm}$ and $5 \mathrm{~mm}$ basipetally (Figure $2 \mathrm{~A}$ ). The entrance diameter was $18 \mathrm{~mm}$. The lower VSD, measuring $5 \mathrm{~mm}$, was located near the crest of the interventricular septum, and the upper VSD, measuring $7 \mathrm{~mm}$, was located close $(2.5 \mathrm{~mm})$ to the aortic end of the septal aneurysm. The distance between the two exits was $4.5 \mathrm{~mm}$.

A $7 \mathrm{~mm}$ and a $6 \mathrm{~mm}$ modified double-disk symmetrical ventricular occluder (lifetech Ltd, Shenzhen, China), similar to the Amplatzer occluder, were used in this procedure. First, the upper exit was crossed from the arterial side using a 6-Fr Launcher Judkins Right 3.5 (JR3.5) guiding catheter with the use of a Terumo wire (Terumo Inc., Japan). The Terumo wire was then exchanged for a $260 \mathrm{~cm}$ long noodle wire (AGA Medical, Golden Valley, MN, USA). Then an arterial-venous wire loop was established from the right femoral artery to the right femoral vein via the defect as previously described. Over the wire a Lifetech delivery sheath was introduced from the femoral vein through the VSD to the LV. Under the guidance
\end{abstract}

of fluoroscopy and TTE, a $7 \mathrm{~mm}$ double-disk symmetrical ventricular occluder was deployed across the defect. The first disc was deployed on the left ventricular side of the septum, and the catheter was pulled back to another disc on the right ventricular side of the defect. Repeated angiogram was then obtained (Figure $2 \mathrm{~B}$ ).

The lower exit then was crossed using the same guiding catheter and wire from the left femoral artery to the left femoral vein. A $6 \mathrm{~mm}$ double-disc symmetrical occluder was deployed across the lower defect overlapping with the first device using the same technique, with excellent results confirmed by LV angiogram (Figure $2 \mathrm{C}$ ) and 2D/3D-TTE (Figures 1 C, D). A continuous pressure recording showed that there was no pressure difference across the right ventricular outflow tract.

After the procedure, electrocardiogram (ECG) showed a normal sinus rhythm. Transthoracic echocardiography after $24 \mathrm{~h}$ showed both devices in good position, with no residual shunt and a lesser degree of tricuspid regurgitation.

The procedure was successful with confirmation by multimodality evaluation including continuous pressure recording, TTE, 3D-TTE, and ECG. During the 12-month follow-up period, there was no heart block, residual shunt, device embolization, infective endocarditis, aortic regurgitation or aggravation of tricuspid regurgitation. At the latest follow-up, the diameters of the left atrium and ventricle had decreased to $25 \mathrm{~mm}$ and $41 \mathrm{~mm}$ respectively.

As the most common congenital heart disease, ventricular septal defect (VSD) accounts for $30 \%$ of all the congenital heart disease. Perimembranous VSD, a defect involving the membranous septum and the adjacent portion of the muscular septum, accounts for about $75-80 \%$ of cases. Although open heart surgery is generally ac-

\section{Corresponding author:}

Sheng-hua Zhou MD, PhD, Department of Cardiology, Second Xiangya Hospital, Central South University, 139 Middle Renmin Road, Changsha, Hunan 410011, China, phone: +86 731 85292012, fax: +86 731 85294038, e-mail: zhoushenghua_guo@163.com

Received: 28.08.2016, accepted: 10.11.2016. 

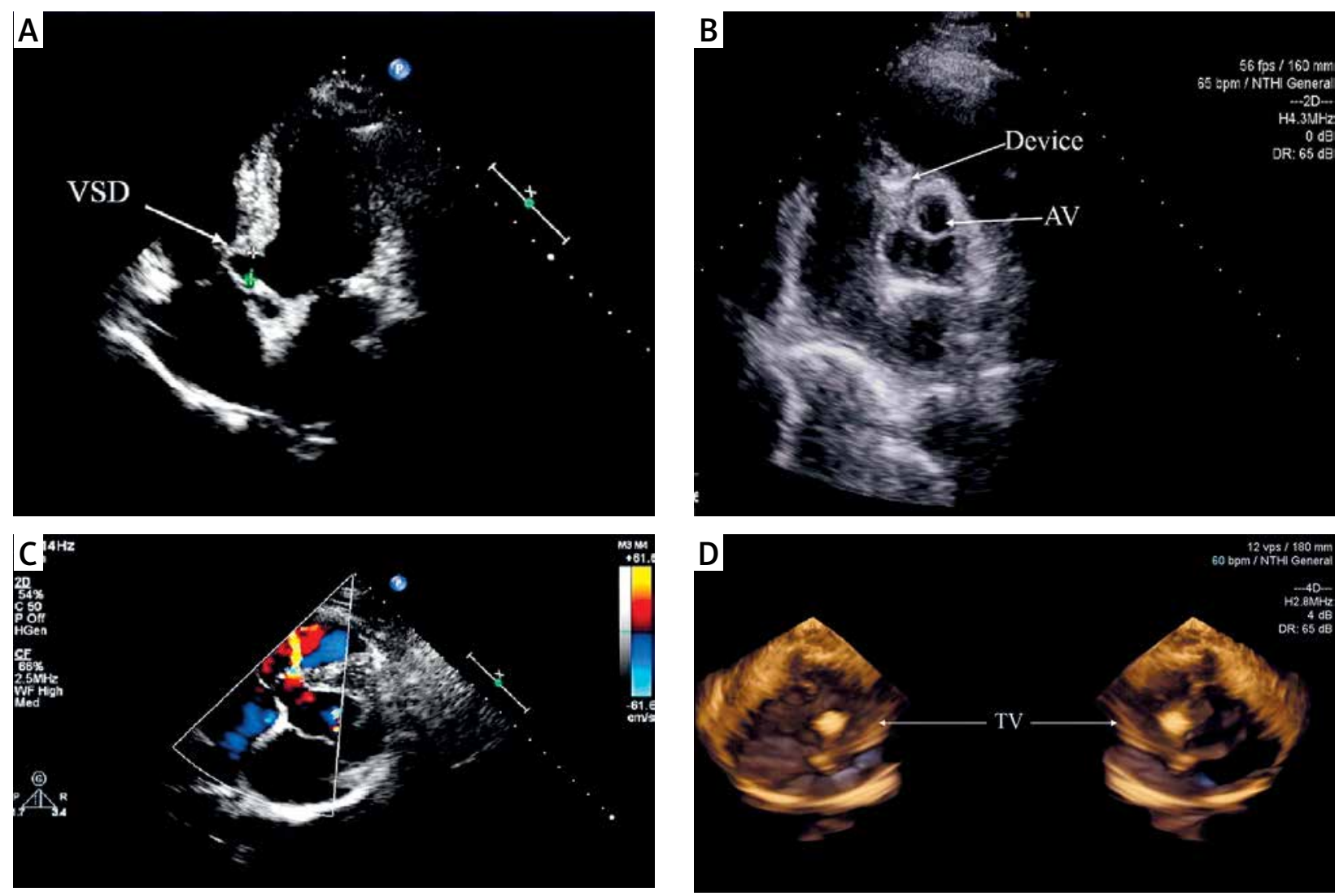

Figure 1. Transthoracic echocardiogram (TTE) showed the ventricular septal defect (VSD): A - two dimensional echocardiography (2DE) obtained in apical four-chamber view showed a perimembranous ventricular septal defect (PmVSD) with a $14 \mathrm{~mm} \times 9 \mathrm{~mm}$ aneurysm. B - Color Doppler flow imaging (CDFI) showed a clear visualization of a $4 \mathrm{~mm}$ left to right shunt through the interventricular septum. C - 2D-TTE showed that both devices were well fixed, overlapping with each other. D - 3D-TTE confirmed that both devices were in a good position without interference with the aortic valve (AV) or tricuspid valve (TV)
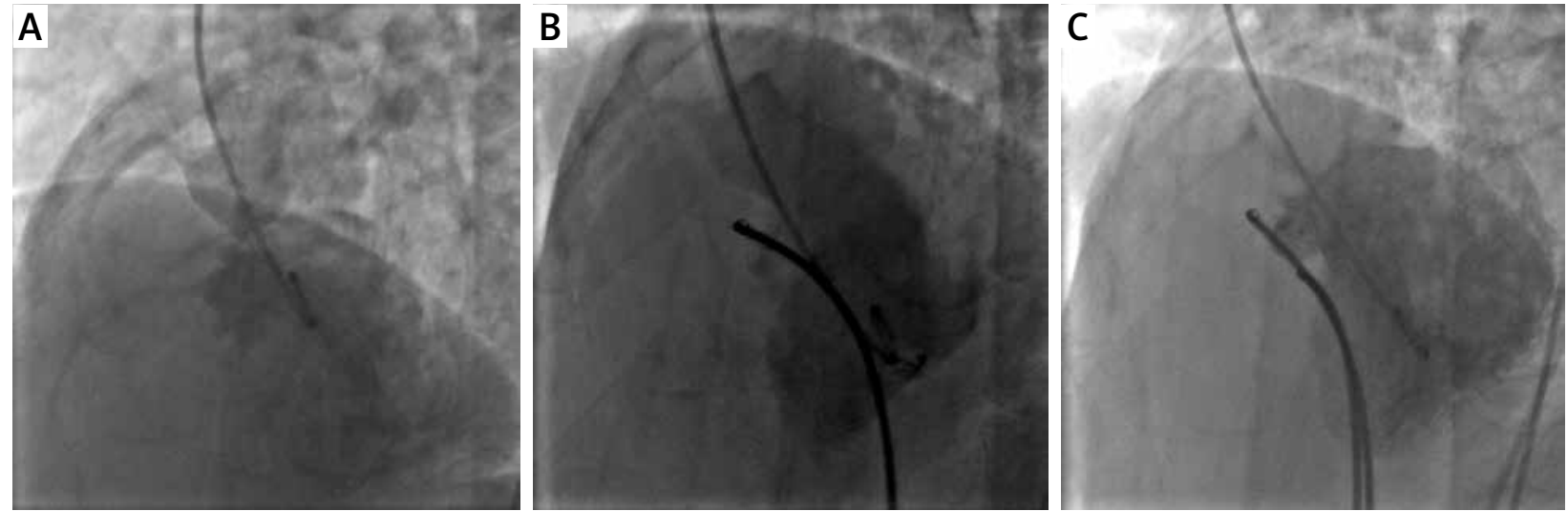

Figure 2. A - Left ventricle (LV) angiogram revealed the PmVSD with a moderate-sized aneurysm of two exits. The entrance diameter was $18 \mathrm{~mm}$. The lower VSD, measuring $5 \mathrm{~mm}$, was located near the crest of the interventricular septum, and the upper VSD, measuring $7 \mathrm{~mm}$, was located close $(2.5 \mathrm{~mm})$ to the aortic end of the septal aneurysm. The distance between the two exits was $4.5 \mathrm{~mm}$. B - Repeated LV angiogram after the deployment of the first device showed that the lower exit was relatively far from the device. C - Repeated LV angiogram after the deployment of both devices showed the complete occlusion of the defect 
knowledged as the standard treatment for PmVSD, with recent increasing use of interventional therapy of VSD and the improvement of the success rate, transcatheter closure has been widely adopted and has been suggested as an attractive alternative to surgery.

In this present case, the aortic angiogram revealed that the defect was perimembranous VSD with a moderate-sized aneurysm which had two exits. Several technical considerations were therefore needed. First, TTE revealed that the aneurysm was relatively long and adjacent to the tricuspid valve. PmVSD often involved the entire membranous septum and adjacent structures such as the aortic valve, tricuspid valve, chordae tendineae, atrioventricular node, and conduction bundle. Color Doppler was therefore especially important for monitoring the occurrence of aortic or tricuspid regurgitation and right ventricular outflow track obstruction during the catheterization procedure. Second, the exits of VSD were relatively far apart, which would probably result in incomplete closure. Incomplete closure often occurs in multihole PmVSD with aneurysm, and occluders may need to be replaced repeatedly during the procedure [1]. Residual shunting due to incomplete closure could cause severe hemolysis requiring repeat intervention or even surgical repair [2]. Third, it was important to choose a suitable occluder. Wu et al. [3] reported an encouraging long-term outcome of 64 patients with multi-hole PmVSDs who underwent a transcatheter closure procedure with a single device using different types of VSD occluders. Retrograde closure of multiple PmVSDs using two ADO II devices was also reported [4]. Although it would make the procedure easier using the arterial route, closure by the antegrade venous approach required a larger distance between the defect and aortic valve. Furthermore, it would probably cause arterial complications and difficulties for achieving a stable position.

To avoid residual shunts and the influence of aortic or tricuspid regurgitation, two double-disc symmetrical occluders were chosen to close the defect because the two exits were far apart and the aneurysm was large enough. Real-time 3D-TTE was performed to further assess the assessment of aortic and tricuspid valve function. Since deploying the devices in the aneurysm would probably cause right ventricular outflow tract obstruction, continuous pressure recording through the right ventricular outflow tract and echocardiography after the procedure were important.

In conclusion, transcatheter closure of multi-hole PmVSDs with aneurysm using more than one device can be performed safely and effectively. Multimodality evaluation, especially the 3D echocardiography which accurately revealed the anatomical structure of cardiac chambers, was important in this procedure for assessing the position of occluders and avoiding an influence on adjacent structures during the procedure.

\section{Conflict of interest}

The authors declare no conflict of interest.

\section{References}

1. Zuo J, Xie J, Yi W, et al. Results of transcatheter closure of perimembranous ventricular septal defect. Am J Cardiol 2010; 106: 1034-7.

2. Szkutnik M, Qureshi SA, Kusa J, et al. Use of the Amplatzer muscular ventricular septal defect occluder for closure of perimembranous ventricular septal defects. Heart 2007; 93: 355-8.

3. Wu H, Qin Y, Zhao X, et al. Transcatheter closure of multi-hole perimembranous VSD with aneurysm: 3-year follow-up study. Clin Res Cardiol 2009; 98: 563-9.

4. Devendran V, Koneti NR, Jesudian V. Transcatheter closure of multiple perimembranous ventricular septal defects with septal aneurysm using two overlapping Amplatzer Duct Occluders II. Pediatr Cardiol 2013; 34: 1963-5. 